\title{
Biomagnetic Measurements for Assessment of Fetal Neuromaturation and Well-Being
}

\author{
Audrius Brazdeikis ${ }^{1}$ and Nikhil S. Padhye ${ }^{2}$ \\ ${ }^{1}$ Department of Physics and Texas Center for Superconductivity, University of Houston \\ ${ }^{2}$ The University of Texas Health Science Center at Houston \\ U. S. A.
}

\section{Introduction}

There has been an explosion of knowledge about the human genome and the complex interplay between the genome and environment that shapes the development of the central nervous system. The development of new quantitative measures that reliably capture early function of the central nervous system is fundamental to assessing the development of the human fetus. Fetal magnetocardiography (fMCG) is a recording of the spatiotemporal magnetic fields created by the fetal cardiac electrical activity that is regulated by the developing central nervous system. It is measured non-invasively by the use of superconducting quantum interference device (SQUID), the most sensitive and stable detector of magnetic flux currently available. The SQUID sensor provides unmatched sensitivity and temporal resolution used for detection of the electromagnetic field perturbation associated with the neuronal currents in the brain, fetal cardiac activity, and the nuclear spin magnetization in ultra-low field nuclear magnetic resonance spectroscopy or in magnetic resonance imaging.

Biomagnetic fMCG measurements remain predisposed to interferences both internal and external to the subject's body, low signal-to-noise ratio, and sensitivity to fetal movements. Successful implementation of the fMCG requires advanced biomagnetometer systems, which usually include arrays of SQUID sensors with automated signal acquisition and control electronics. Specialized software tools are required for signal processing, noise suppression, and artifact removal. In this chapter, we will describe the current status of fMCG applications relevant to their present scientific, technological, and clinical challenges, focusing on fundamental technological breakthroughs in the corresponding fields. Section 2 is devoted to a discussion of SQUID sensor technology, flux transformers, and noise reduction methods. Technological challenges and biomagnetic models that are specific to fetal biomagnetic measurements are described in Section 3.

Considerations in regard to signal processing of fMCG signals and separation of maternal and fetal signals are addressed in Section 4. In contrast to obstetric ultrasound, fMCG permits direct evaluation of the electrophysiological properties of the fetal heart, providing 
information for assessing fetal arrhythmia, prolonged QT-syndrome, and fetal cardiac wave morphology. In addition, fMCG has the precision to accurately quantify beat-to-beat variability such that small, rapid fetal heart rate oscillations associated with respiratory sinus arrhythmia can be detected, quantified, and analyzed to provide a measure of autonomic nervous system control and fetal well-being. Unlike electrocardiography, which is hampered by changing electrical conductance of the fetal-abdominal body during the course of pregnancy, fMCG permits successful recordings from an early stage in the pregnancy. In Section 5, a recent application of fMCG in an unshielded clinical setting is presented that compares spectral and complexity characteristics of heart rate variability in fetuses and in prematurely born neonates of the same age.

\section{Superconducting Sensor Technologies for Weak Magnetic Field Detection}

SQUIDs make use of several physical phenomena including flux-conservation, fluxquantization, and the Josephson effect (Tinkham, 1996). In fact, a SQUID is a superconducting ring, interrupted with one or two weak links called Josephson junctions, which govern the flow of a zero voltage supercurrent. These weak links alter the compensation of the external field by the circulating persistent current, thus making it possible to exploit flux-quantization for measurements of the magnetic fields. Fluxquantization is a unique characteristic of superconductors that provides superconducting sensors with a stability that is possessed by no other magnetic field sensing device. The dc SQUID is generally operated in a finite voltage regime and is effectively a flux-to-voltage transducer characterized by the transfer function which has a periodic sinusoidal function of applied flux with a period equal to one flux quantum $\Phi_{\mathrm{o}}\left(\Phi_{\mathrm{o}} \equiv h / 2 e \approx 2.07 \times 10^{-15} \mathrm{~Wb}\right)$.

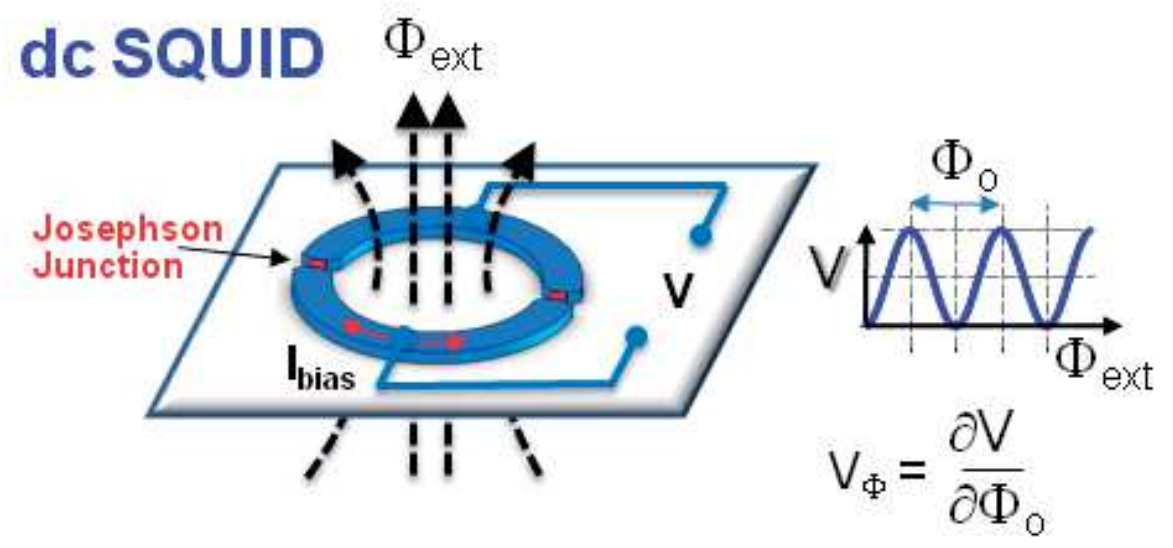

Fig. 1. A schematic illustration of a dc SQUID and its readout characteristic $\left(V-\Phi_{\text {ext }}\right)$. A small change in external magnetic field will produce a change in the readout voltage.

The maximum supercurrent $I_{0}$ through two parallel Josephson junctions in the superconducting ring is modulated periodically by the flux enclosed. When SQUID is biased with a current $I_{\text {bias }}$ slightly higher than $I_{0}$, voltage is developed across the junctions. When 
magnetic flux threading the superconducting ring $\Phi_{\text {ext }}$ is changed, the voltage $V$ across the SQUID oscillates with period of one $\Phi_{\mathrm{o}}$ as shown in Fig. 1 . Any small changes in an external magnetic flux $\Phi_{\text {ext }}$ coupled to the SQUID e.g. due to time varying biomagnetic fields will produce large change in the readout voltage $\delta V=V_{\Phi} \delta \Phi_{\text {ext }}$ where $V_{\Phi}$ is the transfer function. The nonlinear SQUID output can be linearized by using flux-locked loop (FLL) electronics (Drung \& Mück, 2004).

The magnetic field resolution of SQUID sensors is given by their noise performance that is characterized in terms of energy sensitivity, which for dc SQUIDs reach unsurpassed values of $10^{-32} \mathrm{~J} / \mathrm{Hz}$. In the frequency range of interest for biomagnetic measurements $(\mathrm{dc}-100 \mathrm{~Hz})$ noise of commercial SQUID biomagnetometer is typically about $5 \mathrm{fT} \mathrm{rms} \mathrm{Hz}^{-1 / 2}\left(5 \mathrm{fT}=5 \times 10^{-}\right.$ $15 \mathrm{~T}$ ), in part due to magnetic noise generated by electrically conductive radiation-shields of cryogenic dewars (Neonen et al., 1996).

The principal reason for choosing a SQUID sensor for weak magnetic signal detection is because of the tremendous sensitivity in its theoretical and actual performance (e.g. Weinstock, 1996; Weinstock, 2000). The SQUID-based biomagnetic instruments (biomagnetometers) offer the unique combination of sensitivity, wide dynamic range, and frequency response. Measurement of weak magnetic fields generated by nearby biomagnetic sources such as a fetal heart is affected by ambient noise from distant sources, both internal and external to the subject body. Sources of external ambient noise include electrical equipment and instruments connected to the power grid, and various moving magnetic objects such as machinery, cars, and elevators to mention a few. Internal noise sources of biomagnetic origin that are specific to fMCG include the bioelectrical activity of the maternal heart, muscles, gastro-intestinal system, and uterine contractions, especially when close to term. Other specific problems include fetal activity (fetal kicks and movements), maternal breathing moments, and pulsations associated with blood flow (Mosher et al., 1997).

One of the most effective means to reduce the noise detected by a SQUID is through the use of a superconducting flux transformer. All leads of the flux transformer are superconducting, resulting in the total flux linking the SQUID and the coils to be quantized, and therefore stable in time. Any change in the field in the proximity of the pickup coil will modify the total flux in the system, resulting in a change in the persistent current, which in turn will generate an equal but opposite compensating flux such that the total flux trapped is unchanged. This flux change is detected by a SQUID typically placed in a small superconducting (shielded) enclosure far from the measured fields.

The flux transformer coils can have diverse configurations that vary from a single-loop magnetometer to a multi-loop gradiometer as illustrated in Fig. 2. In a single-loop configuration, the flux transformer forms a magnetometer that is sensitive to the normal field component (perpendicular to the plane of the loop). Such a magnetometer is also sensitive to a majority of distant noise sources. A distant noise source is one that is roughly at a distance $r$ of 2 meters or more, as the magnetic field from dipolar sources coupled to a magnetometer falls off as $1 / r^{3}$. The output of two loops can be subtracted, effectively forming a first-gradient field sensor, also referred to as a first-order gradiometer. 


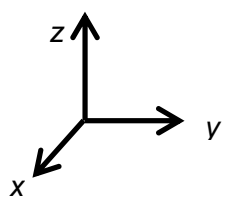

(c)

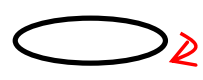

(b)

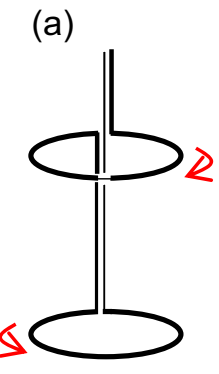

$\partial \mathrm{B}_{\mathrm{z}} / \partial \mathrm{z}$

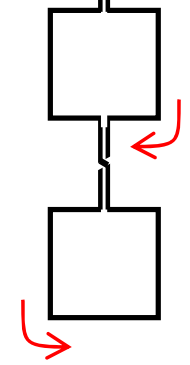

$\partial \mathrm{B}_{\mathrm{x}} / \partial \mathbf{z}$
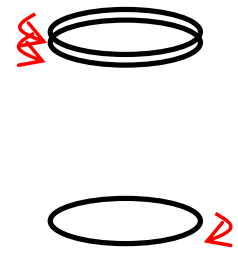

$\partial^{2} \mathrm{~B}_{\mathrm{z}} / \partial \mathrm{z}^{2}$ (d)
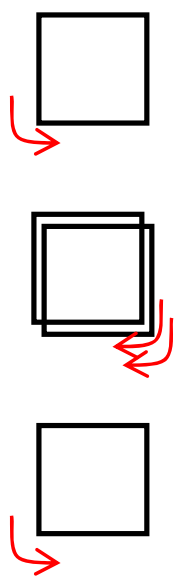

$\partial^{2} B_{x} / \partial z^{2}$

Fig. 2. A schematic illustration of various flux transformer gradiometer configurations: $(a, b)$ first-order gradiometers, (c,d) second-order gradiometers; (b) and (d) show planar configurations.

In most situations, flux transformer output consists of measured biomagnetic signals and ambient noise, and digital filtering methods fail to separate these. The gradiometers, on the other hand, exploit the fact that the gradient of the magnetic field falls off with increasing distance much more rapidly than the uniform field itself. The $n$-th order field gradient falls off as $1 / r^{3+n}$. Thus, gradiometers have a significantly higher sensitivity to nearby sources than distant ones, effectively behaving as spatial high-pass filters that suppress noise from distant sources (Vrba, 1996; Vrba, 2000). An axial gradiometer (Fig. 2a and Fig. 2c) consists of a set of subtracting (wound in the opposite direction) pick-up coils that measure either first- or second-order spatial derivatives $\partial \mathrm{B}_{z} / \partial \mathrm{z}$ or $\partial^{2} \mathrm{~B}_{\mathrm{z}} / \partial \mathrm{z}^{2}\left(\mathrm{~B}_{\mathrm{z}}\right.$ is the $\mathrm{z}$-component of the magnetic field). A planar gradiometer measures either first- or second-order spatial derivatives $\partial \mathrm{B}_{\mathrm{x}} / \partial \mathrm{z}$ or $\partial^{2} \mathrm{~B}_{\mathrm{x}} / \partial \mathrm{z}^{2}$, as shown in Fig. $2 \mathrm{~b}$ and Fig. $2 \mathrm{~d}$.

An alternative approach is to subtract the output signals from two or more gradiometers either electronically or with software to form an electronic (Koch et al., 1993) or a higherorder synthetic gradiometer (Vrba \& Robinson, 2001). Practical gradiometers are characterized by their imbalance (Fagaly, 2006) or unwanted sensitivity to uniform field components and lower-order gradient terms (for second- and higher-order gradiometers). Achieving high gradiometer balance is rather difficult and requires very precise fabrication methods and time-consuming post-assembly balancing using superconducting trim tabs or trim coils. An electronic gradiometer balancing using a reference array of three or more element vector/tensor sensors greatly improves noise rejection by several orders of 
magnitude (Williamson et al., 1985; Matlashov et al., 1989) -all very important for unshielded clinical operation. An axial third-order gradiometer, consisting of two symmetric second-order gradiometers can also be used for improved noise rejection over a wide range of environmental noise conditions (Uzunbajakau et al., 2005).

Other hardware approaches to reduce noise at the location of the sensitive measurements include use of various passive and active shielding methods (Fagaly, 2006). The AC and DC magnetic interferences can be shielded using passive shielding enclosures that employ single- or multi-layer shields of special high permeability and conducting materials. Active shielding strategies employ passive shields together with magnetic field compensation systems, which include separate feedback circuits and sensors that measure the magnetic field and the field gradients. Biomagnetic signals remain predisposed to large interferences, low SNR, and require specialized signal processing tools for noise suppression and artifact removal. Digital signal processing (filtering, averaging) methods and advanced mathematical algorithms that explore linear and nonlinear techniques to reduce noise and various signal artifacts are widely used (Sternickel \& Braginski, 2006). Statistical signal processing techniques (Comani et al., 2004; Hild et al., 2007a; de Araujo et al., 2005; Hild et al., 2007b) such as Independent Component Analysis (ICA) or Blind Source Separation (BSS) are especially useful for extracting fetal MCG data from noisy biomagnetic recordings.

In recent years, development of SQUID instrumentation for acquisition of fetal magnetocardiograms in unshielded environments has become technically feasible (Stolz et al., 2003; Brisinda et al., 2005). Properly designed second-order gradiometers enable detection of weak fetal cardiac signals with signal amplitudes less than $5 \mathrm{pT}$ peak-to-peak in unshielded hospital settings (Brazdeikis et al., 2007).

\section{Factors Affecting the SNR of the Fetal Magnetocardiographic Recordings}

The successful extraction of reliable information such as FHRV from noisy fMCG recordings depends on several factors, including the fetal gestational age and fetal behavioral state. Other factors that also have impact on the signal-to-noise ratio (SNR) are the fetal presentation, placenta location, and maternal bladder volume. As the fetus develops, the fetal myocardium volume increases rapidly from about $20 \mathrm{~mm}$ to $40 \mathrm{~mm}$ (longitudinal axis) during its gestation from 20 to 30 weeks (Chang et al., 1997). Considering fetal myocardium volume as an equivalent current dipole $Q$ inside a spherically symmetric conductor (fetoabdominal body), the increase in active myocardium volume is reflected in increased $Q$, and consequently on the measured magnetic fields (Sarvas, 1987). Kandori et al. (1999a; 1999b) suggested the practical relationship between the strength of an equivalent current dipole $Q$ and gestational age GA as: $Q=18 \mathrm{GA}-295[\mathrm{nAm}]$.

The fetal abdominal depth (source-sensor separation distance) or FAD is a significant factor when estimating the SNR. Based on obstetric ultrasound measurements from 215 pregnant women, Osei \& Faulkner (1999) estimated that the FAD changes with GA as FAD $=0.15$ GA $+5.01[\mathrm{~cm}]$. During weeks 20 to 40 , the FAD increases nearly linearly on average from about $80 \mathrm{~mm}$ to $110 \mathrm{~mm}$. The increase in $Q$ during the same period however is more rapid. There is a consistent improvement in the total SNR as fetal gestational age increases (Fig. 3). 


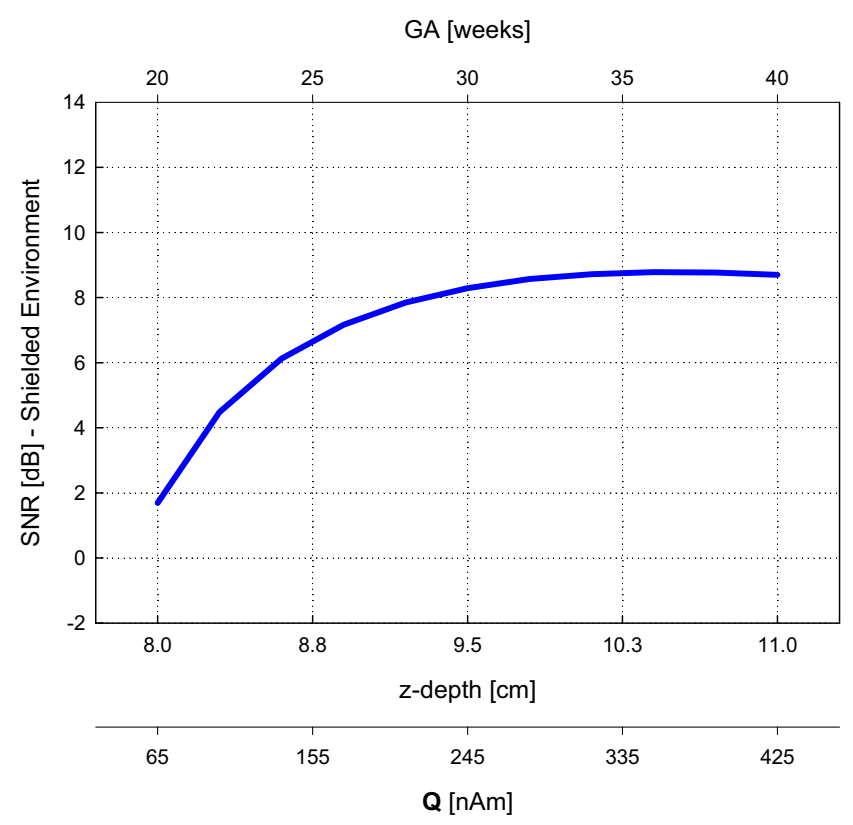

Fig. 3. The monotonic increase in the SNR plotted against the gestational age GA (upper $x$ axis) in shielded environment. The fetal abdominal depth (z-depth) and equivalent current dipole $Q$ values (lower $x$-axes) added for illustration purposes. The biomagnetic model uses an axial second-order-gradiometer pickup, $\partial^{2} \mathrm{~B}_{\mathrm{z}} / \partial \mathrm{z}^{2}$, with a baseline of $70 \mathrm{~mm}$. The flux transformer is assumed to be electronically balanced to $C_{B}=10^{-3}$ (Vázquez-Flores, 2007).

Clinical investigations of fetal heart rate patterns over gestation may be hindered by fetal behavioral state transitions and fetal movements. These behavioral states are distinct and discontinuous modes of autonomic nervous system (ANS) activity. Strong and systematic fetal heart rate changes are accompanied by increased fetal trunk and fetal respiratory movements clearly visible in fMCG recordings (Zhao \& Wakai, 2002; Wakai, 2004). Prolonged heart rate accelerations or decelerations may be associated with directed fetal activity and movement, while irregular heart rate patterns on short timescales may be related to fetal breathing movements (van Leeuwen et al., 2007). Furthermore, fetal activity (fetal kicks and movements) may produce artifacts seen as variation in signal baseline and amplitude, and require reliable techniques to assess fetal gross movements using multichannel SQUID systems (van Leeuwen et al., 2009). 

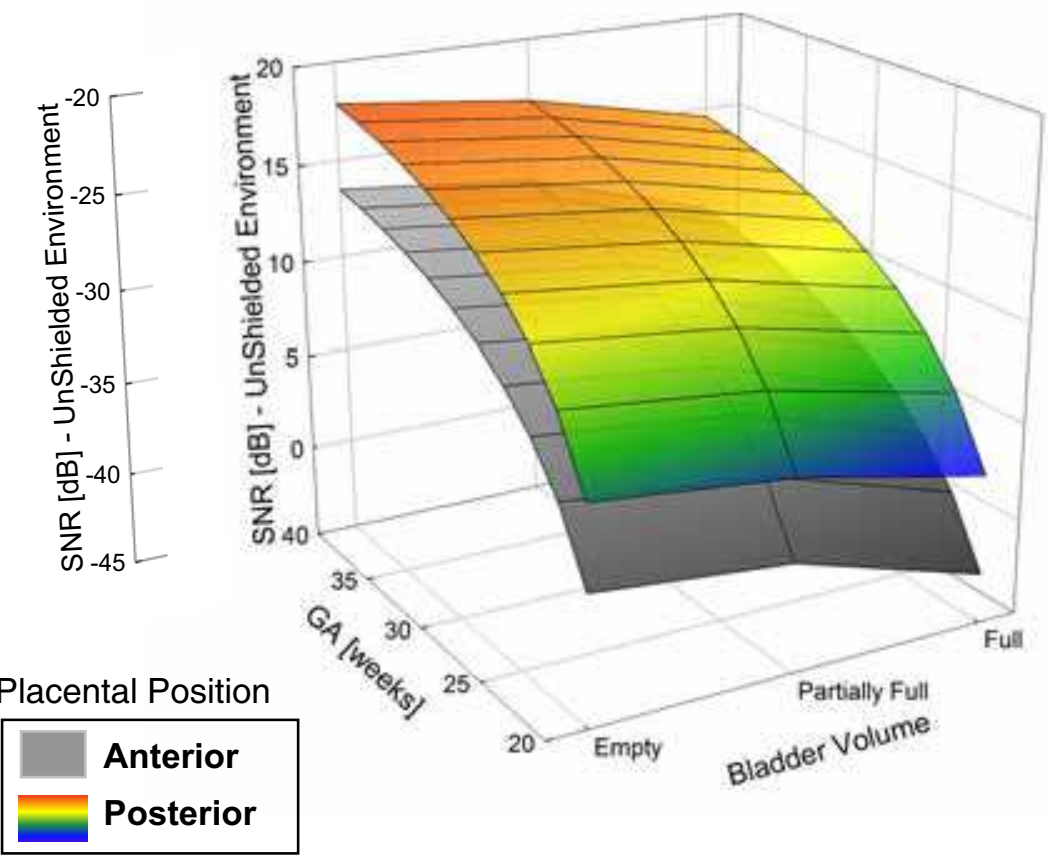

Fig. 4. The SNR calculated as a function of GA and maternal urinary bladder volumes (empty, partially full and full) for posterior and anterior placental positions. Vertical (SNR) axes are shown for shielded and unshielded environments (Vázquez-Flores, 2007).

Ultrasound examination of gravid abdomens (Osei \& Faulkner, 1999) also reveals that the mean FAD is affected by the placenta location and maternal bladder volume. The fetal distance from the anterior surface of a gravid abdomen is shorter for posterior but longer for anterior placental position. When the placenta is located on the anterior uterine wall, the FAD increases by an average of $16 \mathrm{~mm}$. Furthermore, the size of the maternal urinary bladder (full, partially full or empty) also has an effect on the fetal depth and consequently on the SNR. The fetal distance from the anterior surface of a gravid abdomen is shorter for empty bladder but longer for full maternal bladder. When the maternal bladder is full, the FAD increases by an average of $11 \mathrm{~mm}$. Figure 4 shows calculated SNR in shielded and unshielded environments plotted as a function of fetal gestational age and maternal urinary bladder size for anterior and posterior placental positions. The SNR varies by as much as 4 $\mathrm{dB}$ depending on whether the maternal bladder is full or fully distended. In addition, simulation results show a $5 \mathrm{~dB}$ variation in the SNR between posterior and anterior placental positions. The biomagnetic model used for SNR calculations was based on an axial 
second-order-gradiometer pickup, $\partial^{2} \mathrm{~B}_{z} / \partial \mathrm{z}^{2}$, with a baseline of $70 \mathrm{~mm}$. The flux transformer was assumed to be electronically balanced to $C_{B}=10^{-3}$ (Vázquez-Flores, 2007).

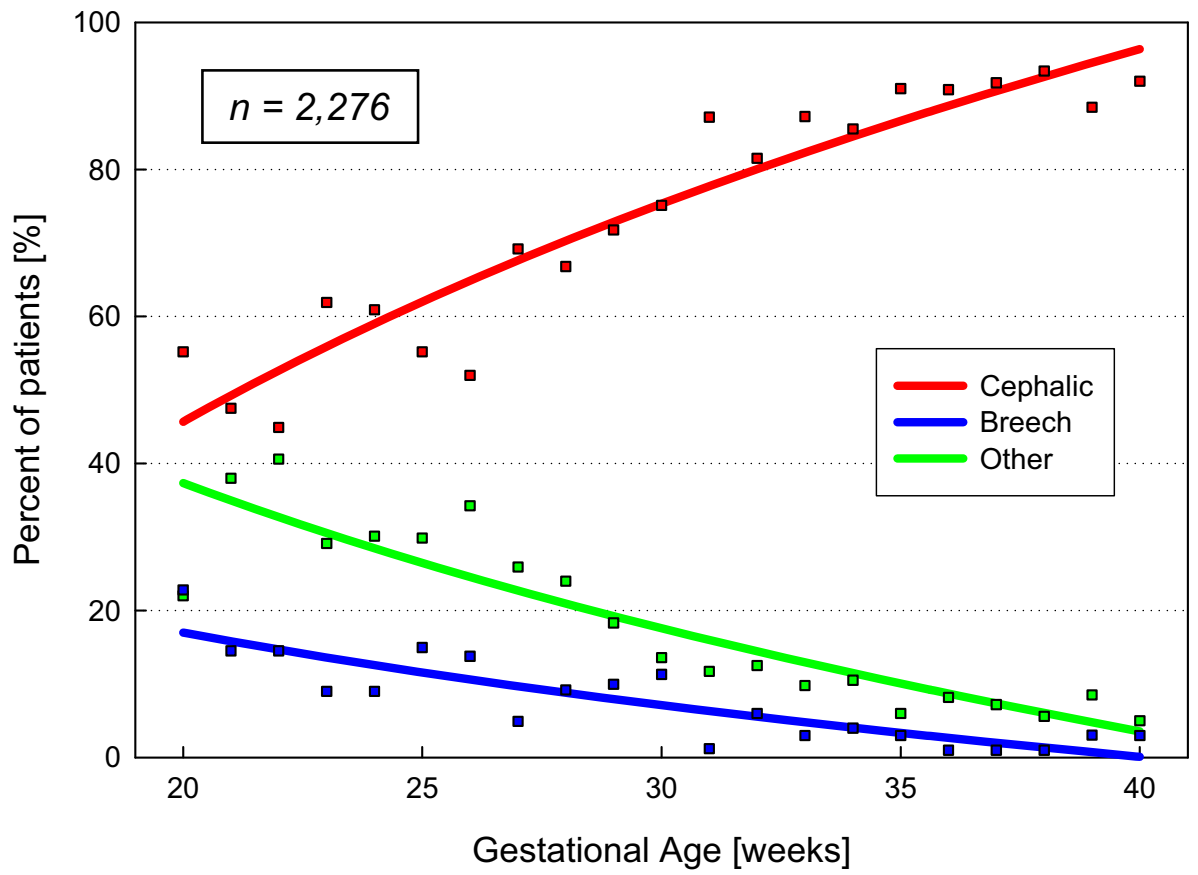

Fig. 5. The prevalence of principal classes of fetal presentation along gestation, as observed in 2,276 subjects by Scheer and Nubar (1976).

Another significant factor affecting fMCG waveform morphology and the SNR is fetal presentation. Fetal presentations are categorized into three principal classes: cephalic, breech, and transverse. Scheer and Nubar (1976) made an exhaustive study of 2,276 pregnant women in which they classified their respective babies into one of the principal presentations. The observed prevalence of fetal presentations in the longitudinal study is summarized in Fig. 5. There is limited published information about the SNR variation and changes in $\mathrm{fMCG}$ waveform morphology for various fetal presentations (Horigome et al. 2006). Although the incidence of cephalic presentation increases with increasing gestational age, the non-cephalic presentation is a common occurrence in early pregnancy when the fetus is highly mobile within a relatively large volume of amniotic fluid. Figure 6 illustrates rather large changes occurring in magnetic field distribution ( $B_{z}$ component) above a gravid abdomen ( $G A=40$ weeks) calculated for cephalic presentation and various axial rotations of fetal body 
Fig. 6. Magnetic field (normal component) distribution above a gravid abdomen (GA=40 weeks) for a cephalic presentation for various fetal body rotations. Biomagnetic modeling data show that up to $30 \%$ signal amplitude variation is possible due to fetal body rotation (Vázquez-Flores, 2007).

\section{Biomagnetic Signal Processing and QRS Detection}

The beat-to-beat changes in fetal heart rate may be masked by incorrect signal processing and QRS detection procedures. Although a wide diversity of QRS detection schemes for electrocardiographic signals have been developed (Köhler et al., 2002; Friesen et al., 1990), automatic QRS techniques specific to fetal magnetocardiograhic signals are rare. A modified Pan-Tompkins QRS detection algorithm has been successfully implemented for automatic QRS detection in normal pregnancies of gestational ages 26-35 weeks (Brazdeikis et al., 2004). The general Pan-Tompkins QRS detection scheme (Pan \& Tompkins, 1985) consists of a band-pass filtering stage, a derivative, squaring and windowing stage, and peak detection and classification stage that matches results from the two previous stages, as illustrated in Fig. 7. Quantitative analysis of fMCG showed excellent QRS detection performance with signal pre-processing and parameter tuning. 


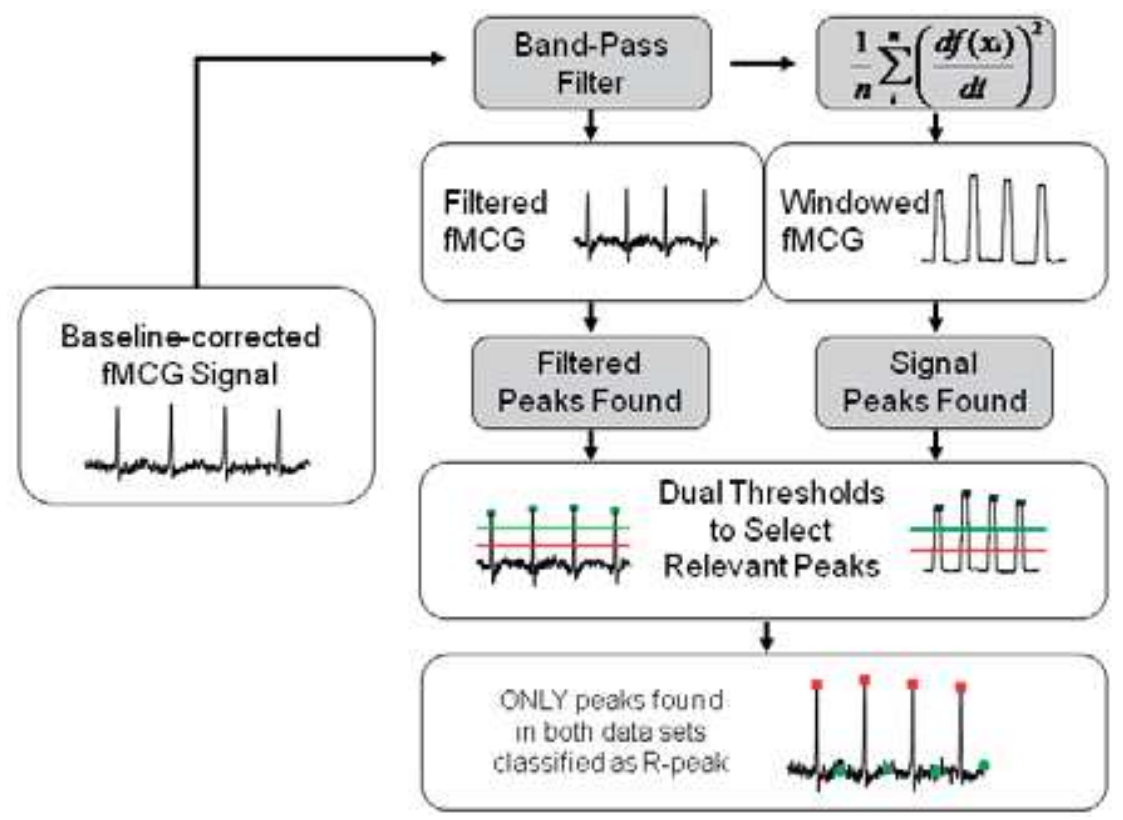

Fig. 7. The general Pan-Tompkins QRS detection scheme adapted for fetal magnetocardiographic signals (Brazdeikis et al., 2004).

When recording fMCG with a second-order gradiometer, the interference from the maternal heart is almost completely absent due to strong spatial high-pass filtering effect. Any remaining maternal MCG signals can be reliably removed by following the cross-correlation procedure illustrated in Fig. 8. In the first step, a classical Pan-Tompkins algorithm was used to extract the maternal RR time series using a reference ECG signal. In the second step, QRS complexes were selectively averaged using a template based on the extracted RR time series. In the final step, the averaged QRS complex was subtracted from the original biomagnetic signal at each location of the maternal QRS, thereby effectively suppressing maternal MCG.

\section{Application of Fetal Magnetocardiography in a Clinical Study}

The application presented in this section utilized clinical data that were collected during two studies of heart rate variability (HRV) at the Texas Medical Center. HRV provides a measure of autonomic nervous system balance, making it possible to gauge maturation of the autonomic nervous system.

In the first study, SQUID technology was used to record magnetocardiograms of fetuses who were 26-35 weeks gestational age. While fMCG recordings are typically done in magnetically shielded environments, the data collected in this study provided evidence that it was possible to obtain fMCG signal in various unshielded hospital settings (Padhye et al., 2004; Verklan et al., 2006; Padhye et al., 2006; Brazdeikis et al., 2007; Padhye et al., 2008). The fMCG signal had sufficiently high signal-to-noise ratio to permit the automated detection of QRS complexes in the fetal magnetocardiograms. 


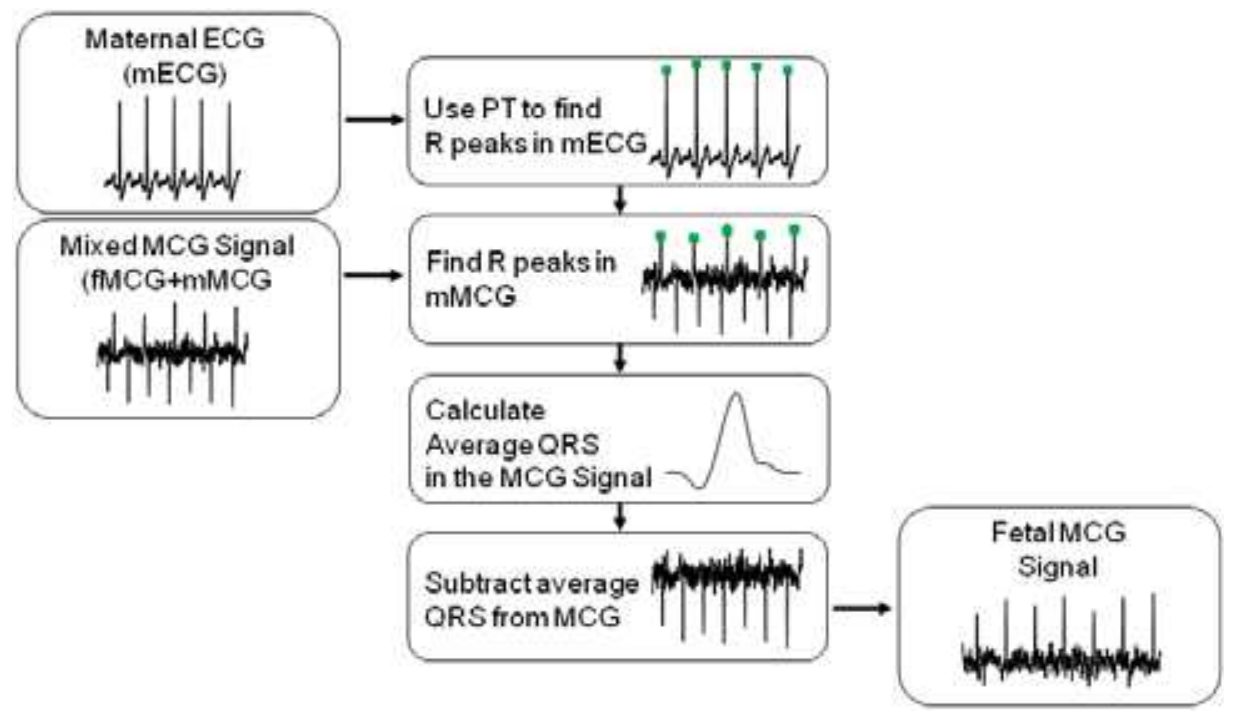

Fig. 8. The Pan-Tompkins QRS detection scheme adapted for removing any interfering maternal signals from fetal magnetocardiograms.

In the second study, electrocardiograms were recorded from prematurely born neonates of 24 to 36 weeks PMA in a neonatal intensive care unit (NICU). The first few minutes of baseline measurements were obtained while the infants were either asleep or lying quietly. The neonates were followed longitudinally and spectral powers of HRV in two frequency bands during the baseline observations were observed to increase as infants matured (Khattak et al., 2007). The increase in HRV is a reflection of the maturing autonomic nervous system. HRV is studied in high and low frequency bands in order to separate the effects of parasympathetic and sympathetic branches of the autonomic nervous system. The question of interest was to compare differences in characteristics of HRV between the fetuses and neonates at closely matched PMA.

HRV was explored in two spectral bands for both fetuses and neonates and modeled statistically to account for the growth of HRV with advancing PMA. Complexity of HRV was studied with multiscale entropy (Costa et al., 2002), which is a measure of irregularity of the fetal and neonatal RR-series. Multiscale entropy is the sample entropy (Richman \& Moorman, 2000) at different timescales of the RR-series, with each scale representing a coarse-graining of the series by that factor. The sample entropy is an inverse logarithmic measure of the likelihood that pairs of observations that match would continue to match at the next observation. Lowered levels of multiscale entropy have been found to be an indicator of fetal distress (Hanqing et al., 2006; Ferrario et al., 2006). Van Leeuwen et al. (1999) reported a closely related quantity, approximate entropy, in fetuses ranging from 16 to 40 weeks and found an increasing trend with age of the fetus. In adult HRV, multiscale entropy has been used successfully to distinguish between beat-to-beat series of normal hearts and those with congestive heart failure and atrial fibrillation (Costa et al., 2002). 
Fractal properties of the RR-series include self-similarity, a property by virtue of which the series appears similar when viewed on different timescales. Self-similarity was quantified for fetal and neonatal RR-series by means of detrended fluctuation analysis (Peng et al., 1994; Goldberger et al., 2000). The presence of log-linear scaling of fluctuations with box sizes provided evidence of self-similar behavior. Two scaling regions were generally present among the fetuses as well as neonates. The scaling in the region with smallest box sizes is closely related to the asymptotic spectral exponent.

\subsection{Data Collection}

Fetal magnetocardiograms were collected at the MSI Center at the Memorial Hermann Hospital in the Texas Medical Center. Seventeen fMCG recordings were obtained from six fetuses with PMA $\geq 26$ weeks. Two fetuses were studied on more than one occasion and the rest were one-time observations. All but one of the recordings were in pairs of consecutive data collection sessions in magnetically shielded and unshielded environments.

As discussed in Section 3, the magnetic signals are largely unaffected by tissue density or conductance variation but fall rapidly with the distance away from the source. This property was used advantageously to filter out interferences arising from the maternal heart, muscle noise, and distant environmental noise sources. A 9-channel SQUID biomagnetometer was employed with second-order gradiometer pick-up coils (see Section 2) that effectively suppressed noise from distant sources while enabling the detection of signals from near sources that generally have stronger gradients at the location of the detector (Brazdeikis et al., 2003). After careful placement of the sensor array over the gravid abdomen it was possible to record fetal magnetocardiograms at several spatial locations largely unaffected by the maternal signal.

Neonatal electrocardiograms were obtained at Children's Memorial Hermann Hospital NICU during the course of a prospective cohort study following 35 very low birth weight $(<1500$ grams) infants over several weeks after admission to the NICU. The neonates ranged from 23 to 38 weeks GA with an entry criterion that required GA at birth $<30$ weeks. A subset was selected of 33 recordings from 13 infants that were relatively healthy and did not require mechanical ventilation. The subset included in the analysis ranged from 24 to 36 weeks GA. Electrocardiograms were recorded from study infants while they were resting for approximately 10 minutes before a blood draw procedure. At the outset, infants were relaxed, eyes were generally closed, and movements were limited to startles and jaw jerks. The Institutional Review Board approved all studies.

\subsection{Measures of HRV}

The fMCG signal was digitized at $1 \mathrm{kHz}$ in each of 9 SQUID channels and signal from the best channel was selected for further analysis. High-frequency noise, baseline drifts, artifacts, and occasionally maternal-MCG were removed using standard techniques of biomagnetic signal processing (see Section 4). The neonatal electrocardiograph signal was similarly digitized at $1 \mathrm{kHz}$. The RR-series for HRV analysis was obtained from either type of signal after implementing a QRS detector using a modified Pan-Tompkins algorithm that was outlined in Section 4. 
The RR-series for each neonatal data set spanned 1000 beats that were part of the baseline recordings, while the full lengths of the fetal RR-series (average of 690 beats per series) were utilized. Far outliers were removed using interquartile range boxes with asymmetric tolerance factors of 3 and 6 on the lower and upper side, respectively, to accommodate strong, natural variability. On average, $0.27 \%$ of data points were deemed far outliers in any data set, while most sets were not affected by far outliers at all.

Since heartbeats are not equispaced in time, the Lomb periodogram (Lomb, 1976) was computed after removing slow trends with a cubic polynomial filter. The Lomb algorithm has some advantages in accuracy of computing the power spectrum for non-uniformly spaced data points over using the fast Fourier transform on an interpolated uniform grid (Laguna \& Moody, 1998; Chang et al., 2001). Spectra were computed on all available segments of RR-series with 192 beats in each segment and skipping forward by 96 beats. Segments had to satisfy a stationarity test that was implemented with the KolmogorovSmirnov test of differences between distributions on sub-segments (Shiavi, 1999). For inclusion, a segment also had to satisfy the condition that the average Nyquist frequency exceed $1.0 \mathrm{~Hz}$, which was the upper limit of our high-frequency band. The band powers were averaged across all segments that passed the criteria. The resulting power spectrum was integrated in the low-frequency (LF) band from 0.05 to $0.25 \mathrm{~Hz}$ and in the highfrequency (HF) band from 0.25 to $1.00 \mathrm{~Hz}$, and band powers were expressed in decibel units with respect to a reference level of $0.02 \mathrm{~ms}^{2}$.
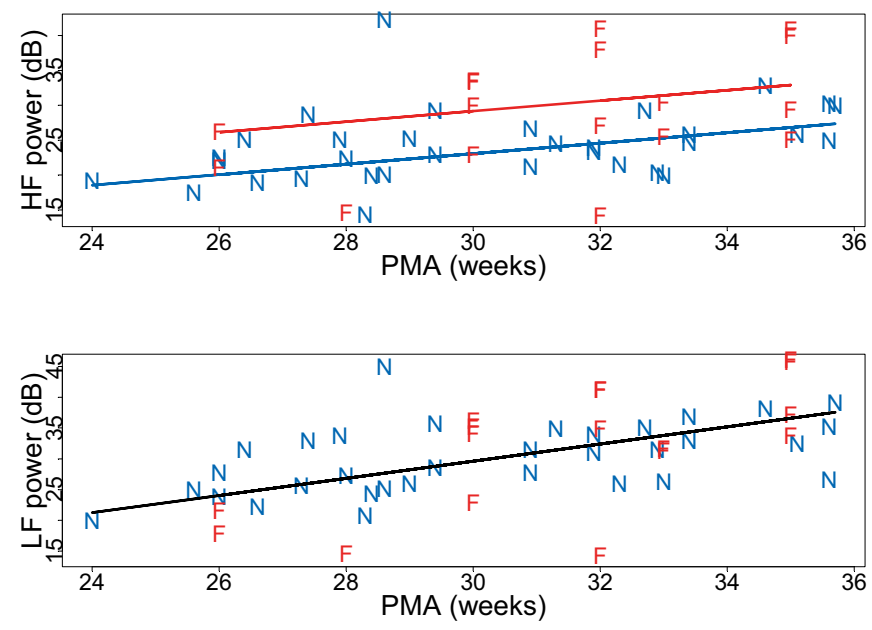

Fig. 9. Top panel depicts growth of HF power with post-menstrual age, differentiated between fetal and neonatal groups. Bottom panel depicts growth of LF power with postmenstrual age. Blue triangles represent fetal observations and red circles are neonatal observations. The lines are model predictions; in the top panel the upper and lower lines are for fetuses and neonates, respectively. 
At any given scale, the sample entropy was computed for pair-wise matching with tolerance set at $20 \%$ of the standard deviation (Richman \& Moorman, 2000). The RR-series was considered to be a point process for the computation of entropy. A self-similar series must necessarily be nonstationary. In first-order detrended fluctuation analysis, the signal at any time is transformed into a signal that has been integrated up to that time instant, ensuring nonstationarity. Fluctuations around linear trends are then computed for varying box sizes. If the resulting logarithm of fluctuations varies linearly with the logarithm of box size, there is evidence of self-similarity. The self-similarity parameter $a$ represents the slope of the linear relationship. It is closely related to the asymptotic spectral exponent and to the Hurst exponent. The slopes of log-linear scaling regions of fluctuations were estimated from regression models. Continuously sliding windows were used in order to minimize estimation error. Since it is important to have precision in the timescales or box sizes in the computation of $a$, the RR-series was uniformly resampled on a grid with $400 \mathrm{~ms}$ spacing between points. The grid spacing corresponds closely to the average RR interval for the sample.

\subsection{Results}

Statistical models were constructed for HF and LF band powers to estimate age related changes and differences between fetal and neonatal HRV with adjustment for age. Robust regression technique was used in order to minimize impact of any large residuals on the model parameters (Yohai et al., 1991). All statistical significances were tested at the $95 \%$ confidence level. The HF power increased $0.75 \pm 0.29 \mathrm{~dB}$ per week in both groups, however the level of HF power was $6.08 \pm 2.11 \mathrm{~dB}$ higher in the fetuses than in the neonates (Fig. 9). The expected value of fetal HF power at 30 weeks PMA was $29.16 \pm 1.80 \mathrm{~dB}$. The LF power increased $1.40 \pm 0.27 \mathrm{~dB}$ per week in fetuses and neonates, but there was neither a significant difference in the LF power levels nor in the rates of growth between the two groups. The expected value of fetal as well as neonatal LF power at 30 weeks PMA was $29.62 \pm 0.87 \mathrm{~dB}$.

The expectation value of the mean RR-interval at 30 weeks PMA was $406.7 \pm 8.6 \mathrm{~ms}$ for a fetus and $13.0 \pm 3.3 \mathrm{~ms}$ lower for a neonate. The mean RR-interval increased by $9.2 \pm 3.0 \mathrm{~ms}$ per week for the fetal group, whereas it declined slightly for the neonates over the age range of the study. The mean heart rate is inversely related to the mean RR-interval.

Estimates of multiscale entropy are progressively less reliable at higher scales. The constraint of series length capped the highest scale at 7. Sample entropy was higher in the fetuses at all scales, as shown in Fig. 10. Statistical models showed differences of $0.24-0.30$, with mean difference of 0.28 . Age-dependent changes in the entropy were not detected at any scale. There was no apparent effect of the magnetic environment, shielded or unshielded, on the shape of the multiscale entropy curves, suggesting that fMCG recordings obtained in unshielded settings are suitable for FHRV studies. The multiscale entropy of a 26 week old fetus showed high entropy at scale 1 and dropped thereafter. This is similar to the relationship of entropy to scale in adults with atrial fibrillation (Costa et al., 2002). The relationship of entropy to scale is reversed in the fetuses 30 weeks GA or older, and resembles that of normal adults (Fig. 11). 


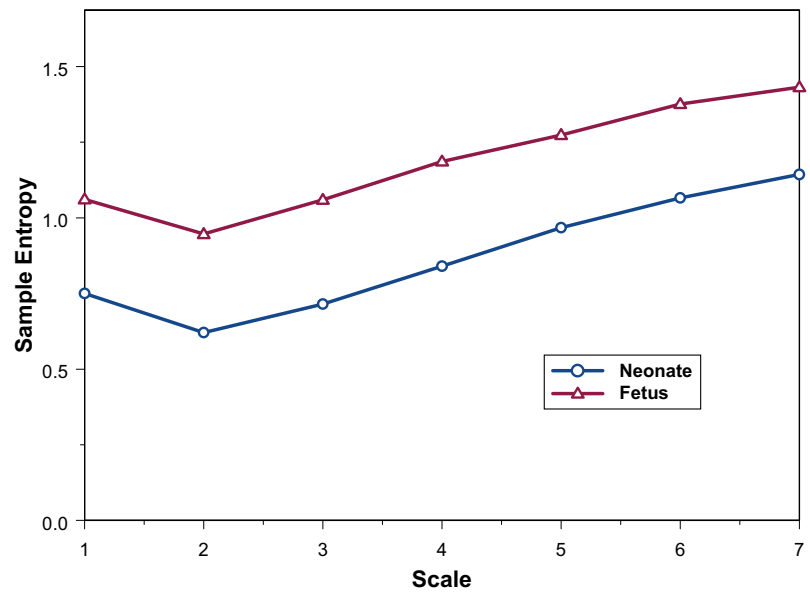

Fig. 10. Average fetal and neonatal entropy vs. scale of RR-series.

Fluctuations scaled linearly with box size on a log-log plot in both fetuses and neonates, indicating the presence of self-similar behavior of the RR-series (Fig. 12). There were typically 2 regions of linear scaling, one at box sizes below 25 (corresponds to timescales below $10 \mathrm{~s}$ ) and a region with reduced slope of scaling at box sizes between 50 and 100 (corresponding to timescales between 20 and $40 \mathrm{~s}$ ). This is in agreement with findings from studies of other fractal properties of fetal HRV (Felgueiras et al., 1998; Kikuchi et al., 2005). Spectral exponents were estimated from the scaling exponent at the fast timescales. The spectral exponent $\beta$ represents the asymptotic slope of the power spectrum $\left(1 / f^{\beta}\right)$. The exponent ranged between 1.3 and 2.6, with a tendency for neonates to exhibit a level that was nearly constant in the age range of the study, while the younger fetuses had a tendency toward lower spectral exponents. The middle $50 \%$ of all exponents were distributed in a narrow band around 2.0, from 1.9 to 2.1 .

\subsection{Implications}

Cardiovascular variance in the HF band is closely related to respiration largely due to the shared control mechanism of the vagus nerve that is part of the parasympathetic nervous system. The decreased level of HRV in neonates in the HF band suggests that the sympathetic/parasympathetic balance of their autonomic nervous system is distinct from that of fetuses at identical post-menstrual ages. It is hypothesized that the physiological stresses of prematurity suppress the activity of the parasympathetic nervous system. Even the healthy "feeder-grower" premature neonate is encumbered with independent respiration and additional metabolic tasks that the fetus is not required to perform. It may be that growth of many systems, including the nervous system, becomes secondary to processes necessary for survival. 


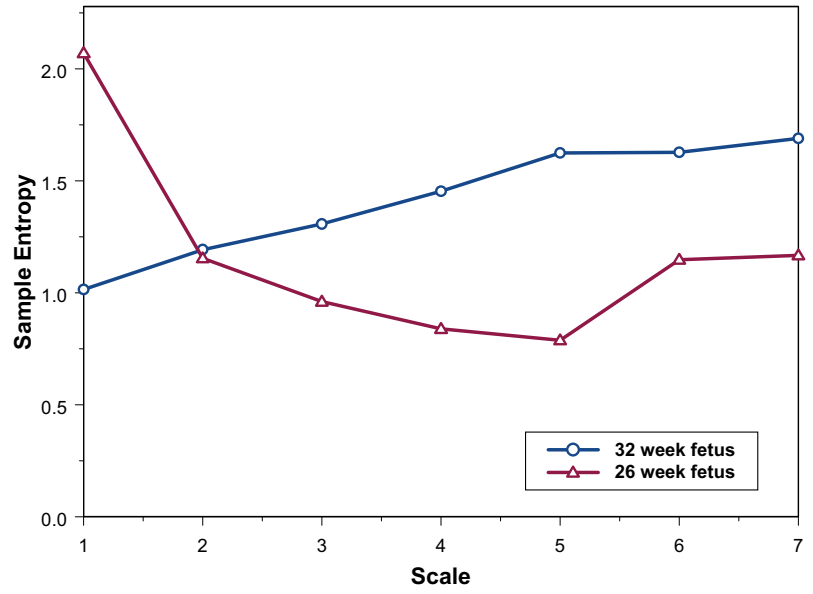

Fig. 11. Multiscale entropy of the heart rate variability of a 26-week fetus and a 32-week fetus show a reversal of relationship between entropy and scale.

The increasing trend of HRV in HF and LF bands in both fetuses and neonates reflects the maturation of parasympathetic and sympathetic nervous systems. The absence of a significant difference in the LF variance between neonates and fetuses suggests that the sympathetic fight-or-flight response is equally well-developed in the two groups.

Entropy of fetal RR-series was higher than the entropy of neonatal RR-series at all scales, which suggests that fetal HRV is more complex and non-repeating than its neonatal counterpart of the same PMA. We investigated the possibility of systematic bias in estimation of entropy due to greater length of the neonatal RR-series, and concluded that stability of estimation was sufficient to discount this possibility. Given the paradigm in the science of complex systems that higher levels of complexity are associated with healthier physiological systems (Goldberger et al., 2002), this may be another indicator that fetal HRV is in a more healthy state than HRV of the prematurely born neonate.

Two regions of scaling were present in the RR-series fluctuations, and there was no discernible difference of scaling regions between fetuses and neonates. Spectral exponents for neonates and fetuses were distributed around the value 2.0, which corresponds to the spectral exponent of a normal diffusion process. This represents a lower level of complexity compared to HRV in healthy adults that exhibits spectral exponents closer to 1 (Yamamoto \& Hughson, 1994). The observed tendencies were for the neonates to have a higher spectral exponent that was steady, while the exponent increased in fetuses with advancing age. However, robust statistical models could not establish the increasing trend in fetuses at the $95 \%$ confidence level. Age-related changes in the scaling exponent were not detected in a larger study of fetal HRV (Lange et al., 2005) suggesting that relative constancy of the spectral exponent may be a property that is shared by fetuses and prematurely born neonates. 


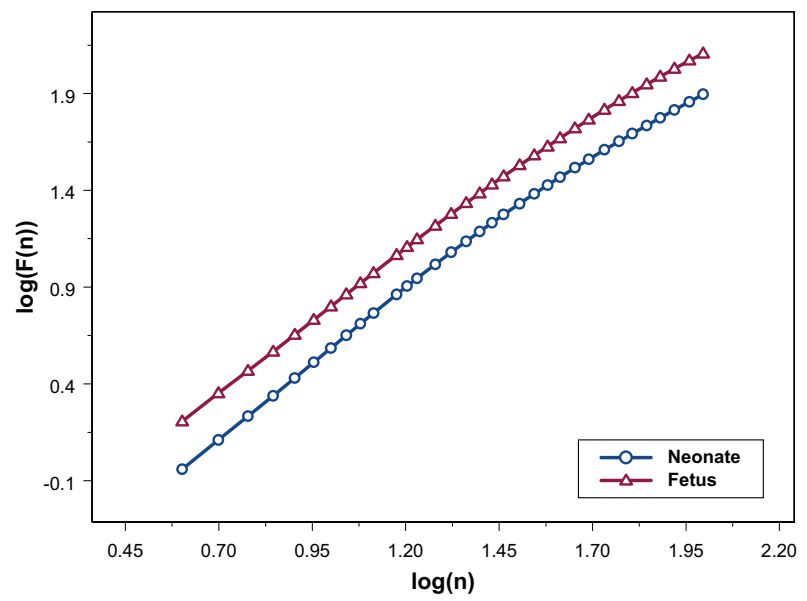

Fig. 12. Fluctuations vs. box size on a log-log scale shows linear relationship below timescale of 10 seconds. Curves represent averages over groups of neonates and fetuses.

The relationship of entropy to scale reversed in observations of fetuses at 26 weeks and 30 weeks gestational age, which may be indicative of a critical stage of maturation in the autonomic nervous system that controls their heart rate variability. This pilot study is limited by the sample size. More data is required, especially for fetuses younger than 30 weeks gestational age, before a more confident conclusion can be drawn. Spectral as well as complexity measures were computed from recordings in the unshielded environment that did not differ appreciably from corresponding measures computed from recordings in magnetically shielded rooms.

\section{Conclusion}

Fetal magnetocardiography offers direct evaluation of the electrophysiological properties of the fetal heart from an early stage of fetal development. It offers potentially more accurate examination of beat-to-beat intervals than does fetal ultrasound or fetal ECG. At present its wide clinical adoption is limited since it requires expensive magnetically shielded rooms. Recent successes in recording fetal magnetocardiograms with relatively small systems outside the shielded environment are a promising development. Application of the technical and computational tools was illustrated in a clinical study that compared spectral and complexity properties of heart rate variability in fetuses and age-matched, prematurely born neonates. Future work in fetal magnetocardiography is likely to focus on development of technology that is affordable for wide clinical deployment at the bedside and that is supported by diagnostics of fetal neuromaturation and stress based on measures of heart rate variability. 


\section{References}

Brazdeikis, A.; Xue, Y. Y. \& Chu, C. W. (2003). Non-invasive assessment of the heart function in unshielded clinical environment by SQUID gradiometry. IEEE Trans. Appl. Supercond., 13, pp 385-388

Brazdeikis, A.; Guzeldere, A. K.; Padhye, N. S. \& Verklan, M. T. (2004). Evaluation of the performance of a QRS detector for extracting the heart interbeat RR time series from fetal magnetocardiography recordings, Proc. 26th Ann. Intl. Conf. IEEE Eng. in Med. and Biol. Soc., pp. 369-372, San Francisco, CA, USA

Brazdeikis, A.; Vázquez-Flores, G. J.; Tan, I. C.; Padhye, N. S. \& Verklan, M. T. (2007). Acquisition of fetal magnetocardiograms in an unshielded hospital setting. IEEE Transactions on Applied Superconductivity, 17, 2, pp. 823-826

Brisinda, D.; Comani, S.; Meloni, A. M.; Alleva, G.; Mantini, D. \& Fenici, R. (2005). Multichannel mapping of fetal magnetocardiogram in an unshielded hospital setting. Prenatal Diagnosis, 25, pp. 376-382

Chang, F. M.; Hsu, K. F.; Ko, H. C.; Yao, B. L.; Chang, C. H.; Yu, C. H.; Liang, R. I. \& Chen, H. Y. (1997). Fetal heart volume assessment by three-dimensional ultrasound. Ultrasound. Obstet. Gynecol., 9, pp. 42-48

Chang, K. L.; Monahan, K. J.; Griffin, M. P.; Lake, D. \& Moorman, J. R. (2001). Comparison and clinical application of frequency domain methods in analysis of neonatal heart rate time series. Ann. Biomed. Eng., 29, pp. 764-774

Comani, S.; Mantini, D.; Alleva, G.; Di Luzio, S. \& Romani, G. L. (2004). Fetal magnetocardiographic mapping using independent component analysis. Physiol. Meas., 25, 6, pp. 1459-1472

Costa, M.; Goldberger, A. L. \& Peng, C. K. (2002). Multiscale entropy analysis of complex physiologic time series. Phys. Rev. Lett., 89, 068102

De Araujo, D. B.; Barros, A. K.; Estombelo-Montesco, C.; Zhao, H.; da Silva Filho, A. C.; Baffa, O.; Wakai, R.; Ohnishi, N. (2005). Fetal source extraction from magnetocardiographic recordings by dependent component analysis. Phys. Med. Biol., 50, 19, pp. 4457-4464

Drung, D. \& Mück, M. (2004). SQUID electronics, In: The SQUID Handbook, Clarke, J. \& Braginski, A. I. (Eds.), pp. 127-170, Wiley-VCH

Fagaly, R. L. (2006). Superconducting quantum interference device instruments and applications. Rev. Sci. Instrum., 77, 101101-45

Felgueiras, C. S.; de Sa' Marques, J. P.; Bernardes, J. \& Gama, S. (1998). Classification of foetal heart rate sequences based on fractal features. Med. Biol. Eng. Comput., 36, pp. 197-201

Ferrario, M.; Signorini, M. G.; Magenes, G. \& Cerutti, S. (2006). Comparison of entropybased regularity estimators: Application to the fetal heart rate signal for the identification of fetal distress. IEEE Transactions on Biomedical Engineering, 53, pp. 119-125

Friesen, G. M.; Jannett, T. C.; Jadallah, M. A.; Yates, S. L.; Quint, S. R. \& Nagle, H. T. (1990). A comparison of the noise sensitivity of nine QRS detection algorithms. IEEE Trans. Biomed. Eng., 37, pp. 85-98

Goldberger, A. L.; Amaral, L. A. N.; Glass, L.; Hausdorff, J. M.; Ivanov, P. C.; Mark, R. G.; Mietus, J. E.; Moody, G. B.; Peng, C. K. \& Stanley, H. E. (2000). PhysioBank, PhysioToolkit, and PhysioNet: Components of a new research resource for complex 
physiologic signals. Circulation, 101, 23, pp. e215-e220 [Circulation Electronic Pages; http://circ.ahajournals.org/cgi/content/full/101/23/e215]

Goldberger, A. L.; Peng, C. K. \& Lipsitz, L. A. (2002). What is physiologic complexity and how does it change with aging and disease? Neurobiol. Aging, 23, pp. 23-26

Hanqing, C.; Lake, D. E.; Ferguson, J. E.; Chisholm, C. A.; Griffin, M. P. \& Moorman, J. R. (2006). Toward quantitative fetal heart rate monitoring. IEEE Transactions on Biomedical Engineering, 53, pp. 111-118

Hild, K. E.; Alleva, G.; Nagarajan, S. \& Comani, S. (2007a). Performance comparison of six independent components analysis algorithms for fetal signal extraction from real fMCG data. Phys. Med. Biol., 52, pp. 449-462

Hild, K. E.; Attias, H. T.; Comani, S. \& Nagarajan, S. S. (2007b). Fetal cardiac signal extraction from magnetocardiographic data using a probabilistic algorithm. Signal Proc., 87, pp. 1993-2004

Horigome, H.; Ogata, K.; Kandori, A.; Miyashita, T.; Takahashi-Igari, M.; Chen, Y. J.; Hamada, H. \& Tsukada, K. (2006). Standardization of the PQRST waveform and analysis of arrhythmias in the fetus using vector magnetocardiography. Pediatr. Res., 59, pp. 121-125

Kandori, A.; Miyashita, T.; Tsukada, K.; Horigome, H.; Asaka, M.; Shigemitsu, S.; Takahashi, M. I.; Terada, Y. \& Mitsui, T. (1999a). Sensitivity of foetal magnetocardiograms versus gestation week. Med. Biol. Eng. Comput., 37, pp. 545-548

Kandori, A.; Miyashita, T.; Tsukada, K.; Horigome, H.; Asaka, M.; Shigemitsu, S.; Takahashi, M.; Terada, Y.; Mitsui, T. \& Chiba, Y. (1999b). A vector fetal magnetocardiogram system with high sensitivity. Rev. Sci. Instrum., 70, pp. 4702-4705

Khattak, A. Z.; Padhye, N. S.; Williams, A. L.; Lasky, R. E.; Moya, F. R. \& Verklan, M. T. (2007). Longitudinal assessment of heart rate variability in very low birth weight infants during their NICU stay. Early Hum. Dev., 83, pp. 361-366

Kikuchi, A.; Unno, N.; Horikoshi, T.; Shimizu, T.; Kozuma, S. \& Taketani, Y. (2005). Changes in fractal features of fetal heart rate during pregnancy. Early Hum. Dev., 81, pp. 655661

Köhler, B.-U.; Hennig, C. \& Orglmeister, R. (2002). The principles of software QRS detection. IEEE Eng. Med. Biol. Mag., 21, pp. 42-57

Koch, R. H.; Rozen, J. R.; Sun, J. Z. \& Gallagher, W. J. (1993). Three SQUID gradiometer. Appl. Phys. Lett., 63, pp. 403-405

Laguna, P. \& Moody, G. B. (1998). Power spectral density of unevenly sampled data by least-square analysis: Performance and application to heart rate signals. IEEE Trans. Biomed. Eng., 45, pp. 698-715

Lange, S.; Van Leeuwen, P.; Geue, D.; Cysarz, D. \& Grönemeyer, D. (2005). Application of DFA in fetal heart rate variability. Biomedizinsiche Technik, 50, suppl. 1, pp. 14811482

Lomb, N. R. (1976). Least-squares frequency analysis of unequally spaced data. Astrophys. and Space Sci., 39, pp. 447-462

Matlashov, A.; Zhuravlev, Y.; Lipovich, A.; Alexandrov, A.; Mazaev, E.; Slobodchikov, V. \& Washiewski, O. (1989). Electronic noise suppression in multi-channel neuromagnetic system, In: Advances in Biomagnetism, Williamson, S. J.; Hoke, M.; Stroink, G. \& Kotani, M. (Eds.), pp. 7725-7728, Plenum Press, New York 
Mosher, J. C.; Flynn, E. R.; Quinn, A.; Weir, A.; Shahani, U.; Bain, R. J. P.; Maas, P. \& Donaldson, G. B. (1997). Fetal magnetocardiography: methods for rapid data reduction. Rev. Sci. Instrum., 68, pp. 1587-1595

Neonen, J.; Montonen, J. \& Katila, T. (1996). Thermal noise in biogmagnetic measurements. Rev. Sci. Instrum., 67, pp. 2397-2405

Osei, E. K. \& Faulkner, K. (1999). Fetal position and size data for dose estimation. Br. J. Radiol., 72, pp. 363-370

Padhye, N. S.; Brazdeikis, A. \& Verklan, M. T. (2004). Monitoring fetal development with magnetocardiography, Proc. 26th Ann. Intl. Conf. IEEE Eng. in Med. and Biol. Soc., pp. 3609-3610, San Francisco, CA, USA

Padhye, N. S.; Brazdeikis, A. \& Verklan, M. T. (2006). Change in complexity of fetal heart rate variability, Proc. 28th Ann. Intl Conf. IEEE Eng. in Med. and Biol. Soc., pp. 17961798, New York City, NY, USA

Padhye, N. S.; Verklan, M. T.; Brazdeikis, A.; Williams, A. L.; Khattak, A. Z. \& Lasky, R. E. (2008). A comparison of fetal and neonatal heart rate variability at similar postmenstrual ages, Proc. 30th Ann. Intl Conf. IEEE Eng. in Med. and Biol. Soc., pp. 28012804, Vancouver, BC, Canada

Pan J. \& Tompkins, W. J. (1985). A real-time QRS detection algorithm. IEEE Trans. Biomed. Eng., 32, pp. 230-236

Peng, C. K.; Buldyrev, S. V.; Havlin, S.; Simons, M.; Stanley, H. E. \& Goldberger, A. L. (1994). Mosaic organization of DNA nucleotides. Phys. Rev. E, 49, pp. 1685-1689

Richman, J. S. \& Moorman, J. R. (2000). Physiologic time series analysis using approximate entropy and sample entropy. Am. J. Physiol., 278, pp. 2039-2049

Sarvas, J. (1987). Basic mathematical and electromagnetic concepts of the biomagnetic inverse problem. Phys. Med. Biol., 32, pp. 11-22

Scheer, K. \& Nubar, J. (1976). Variation of fetal presentation with gestational age. Am. J. Obstet. Gynecol., 125, pp. 269-270

Shiavi, R. (1999). Introduction to applied statistical signal analysis, 2nd ed., Academic Press, San Diego

Sternickel, K. \& Braginski, A. I. (2006). Biomagnetism using SQUIDs: status and perspectives. Supercond. Sci. Technol., 19, pp. S160-S171

Stolz, R.; Bondarenko, N.; Zakosarenko, V.; Schulz, M. \& Meyer, H. G. (2003). Integrated gradiometer-SQUID system for fetal magneto-cardiography without magnetic shielding. Superconductivity Science Technologies, 16, pp. 1523-1527

Tinkham, M. (1996). Introduction to Superconductivity, McGraw-Hill, New York

Uzunbajakau, S. A.; Rijpma, A. P.; ter Brake, H. J. M. \& Peters, M. J. (2005). Optimization of a third-order gradiometer for operation in unshielded environments. IEEE Trans. Appl. Supercond., 15, pp. 3879-3885

Van Leeuwen, P.; Lange, S.; Bettermann, H.; Grönemeyer, D. \& Hatzmann, W. (1999). Fetal heart rate variability and complexity in the course of pregnancy. Early Hum. Dev., 54, pp. 259-269

Van Leeuwen, P.; Cysarz, D.; Lange, S. \& Geue, D. (2007). Quantification of fetal heart rate regularity using symbolic dynamics. Chaos, 17, 015119-9

Van Leeuwen, P.; Geue, D.; Lange, S. \& Groenemeyer, D. (2009). Analysis of fetal movement based on magnetocardiographically determined fetal actograms and fetal heart rate 
accelerations, In: ECIFMBE 2008, IFMBE Proceedings Vol. 22, Vander Sloten, J.; Verdonck, P.; Nyssen, M. \& Haueisen, J. (Eds.), pp. 1386-1389, Springer, Berlin

Vázquez-Flores, G. J. (2007). A realistic biomagnetic model for optimized acquisition of fetal magnetocardiograms in unshielded clinical settings, Thesis, University of Houston

Verklan, M. T.; Padhye, N. S. \& Brazdeikis, A. (2006). Analysis of fetal heart rate variability obtained by magnetocardiography. J. Perinat. Neonat. Nurs., 20, pp. 343-348

Vrba, J. (1996). SQUID gradiometers in real environments, In: SQUID Sensors: Fundamentals, Fabrication and Applications, Weinstock, H. (Ed.), pp. 117-178, Kluwer Academic Publishers, Dordrecht

Vrba, J. (2000). Multichannel SQUID biomagnetic systems, In: Applications of Superconductivity, Weinstock, H. (Ed.), pp. 61-138, Kluwer Academic Publishers, Dordrecht

Vrba, J. \& Robinson, S. E. (2001). Signal processing in magnetoencephalography. Methods, 25, pp. 249-271

Wakai, R. T. (2004). Assessment of fetal neurodevelopment via fetal magnetocardiography. Experimental Neurology, 190, Suppl. 1, pp. S65-S71

Weinstock, H. (1996). SQUID Sensors: Fundamentals, Fabrication and Applications, Kluwer Academic Publishers, Dordrecht

Weinstock, H. (2000). Applications of Superconductivity, Kluwer Academic Publishers, Dordrecht

Williamson, S. J.; Pellizone, M.; Okada, Y.; Kaufman, L.; Crum, D. B. \& Marsden, J. R. (1985). Five channel SQUID installation for unshielded neuromagnetic measurements, In: Biomagnetism: Applications and Theory, Weinberg, H.; Stroink, G. \& Katila T. (Eds.), pp. 46-51, Pergamon Press, New York

Yamamoto, Y. \& Hughson, R. L. (1994). On the fractal nature of heart rate variability in humans: effects of data length and beta-adrenergic blockade. Am. J. Physiol., 266, pp. R40-R49

Yohai, V.; Stahel, W. A. \& Zamar, R. H. (1991). A procedure for robust estimation and inference in linear regression, In: Directions in Robust Statistics and Diagnostics, Part II, Stahel W. A. \& Weisberg, S. W. (Eds.), Springer-Verlag, Berlin

Zhao, H. \& Wakai, R. T. (2002). Simultaneity of foetal heart rate acceleration and foetal trunk movement determined by foetal magnetocardiogram actocardiography. Phys. Med. Biol., 47, pp. 839-846 


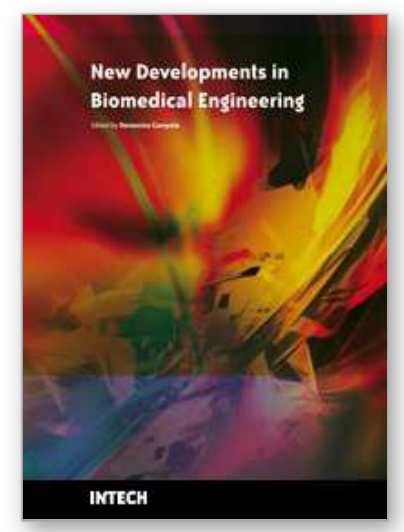

\author{
New Developments in Biomedical Engineering \\ Edited by Domenico Campolo
}

ISBN 978-953-7619-57-2

Hard cover, 714 pages

Publisher InTech

Published online 01, January, 2010

Published in print edition January, 2010

Biomedical Engineering is a highly interdisciplinary and well established discipline spanning across engineering, medicine and biology. A single definition of Biomedical Engineering is hardly unanimously accepted but it is often easier to identify what activities are included in it. This volume collects works on recent advances in Biomedical Engineering and provides a bird-view on a very broad field, ranging from purely theoretical frameworks to clinical applications and from diagnosis to treatment.

\title{
How to reference
}

In order to correctly reference this scholarly work, feel free to copy and paste the following:

Audrius Brazdeikis and Nikhil S. Padhye (2010). Biomagnetic Measurements for Assessment of Fetal Neuromaturation and Well-Being, New Developments in Biomedical Engineering, Domenico Campolo (Ed.), ISBN: 978-953-7619-57-2, InTech, Available from: http://www.intechopen.com/books/new-developments-inbiomedical-engineering/biomagnetic-measurements-for-assessment-of-fetal-neuromaturation-and-well-being

\section{INTECH}

open science | open minds

\section{InTech Europe}

University Campus STeP Ri Slavka Krautzeka 83/A 51000 Rijeka, Croatia Phone: +385 (51) 770447

Fax: +385 (51) 686166 www.intechopen.com

\section{InTech China}

Unit 405, Office Block, Hotel Equatorial Shanghai No.65, Yan An Road (West), Shanghai, 200040, China 中国上海市延安西路65号上海国际贵都大饭店办公楼405单元

Phone: +86-21-62489820

Fax: +86-21-62489821 
(C) 2010 The Author(s). Licensee IntechOpen. This chapter is distributed under the terms of the Creative Commons Attribution-NonCommercial-ShareAlike-3.0 License, which permits use, distribution and reproduction for non-commercial purposes, provided the original is properly cited and derivative works building on this content are distributed under the same license. 